\title{
Cardiac Finding in CKD Patient in Geriatric Population
}

\author{
Bharti $T^{1}$, Dr. Punit $\mathbf{G}^{2}$ \\ ${ }^{1}$ Fellowship in Advance Clinical Research, ${ }^{2}$ Professor Nephrology Unit, \\ Department of medicine, Dr. Bhimrao Ambedkar Hospital, Raipur, Chhattisgarh, India
}

\begin{abstract}
Geriatric population is increasing worldwide due to increased life expectancy. Therefore the present study was conducted to see the cardiac finding in chronic kidney disease (CKD) in them. Chronic kidney disease (CKD) patients are highly prone to cardiovascular disease for a number of reasons. At the time of starting renal replacement treatment, their cardiovascular condition is already severely compromised, suggesting that cardiovascular risk factors begin to operate very early in the progression of CKD. Moreover, those patients reaching end-stage renal disease without cardiovascular abnormalities have a high probability of developing de novo cardiovascular disease. This study discusses the microscopic and macroscopic changes in cardiovascular structure, function, protective systems, and problem associated with aging in CKD patients.
\end{abstract}

Keyword: geriatric; elderly; anaemia; cardiovascular disease; chronic kidney disease; dyslipidaemia; hypertension.

Materials and Method: This retrospective study included 100 patients above the age of 50 years, in the Department of Medicine under nephrology unit, Dr. Bhimrao Ambedkar Hospital Raipur (C.G.), during the period of February 2018 - July 2018 were selected in this prospective study. Patients were analysed based on Clinical evaluation and relevant investigations including echocardiography and classified under different categories of renal diseases.

Results: Mean age of study population was $56.62 \pm 5.44$ years. Breathing probleme is the commonest symptom 89\%, Pedal edema $79 \%$, Anemia shows in 93\%, Heart failure with reduced ejection fraction (HFrEF) and Heart Failure with preserved ejection fraction (HFpEF) were present in $32 \%$ and $70 \%$ ) respectively. Left ventricular (LV) hypertrophy was present in $49 \%$. Pericardial effusion was present in $18 \%$. Complete heart block was present in 5\%. Pulmonary hypertension $(\mathrm{PH})$ was present in $42 \%$.

Conclusion: Only very few studies are available on the spectrum of AKI in older age group. The incidence of AKI is increasing, especially among the elderly. Studies reveal that the elderly suffer higher morbidity and mortality from AKI.

\section{Background}

Renal diseases are one of the common diseases causing high morbidity and mortality in otherwise asymptomatic individuals. The spectrum of renal diseases varies significantly in different parts of the world and is influenced by geographical, environmental and socioeconomic factors in that region. In addition, the spectrum also varies depending upon the population group being studied: community, outdoor patients or inpatients of a general/tertiary care hospital.

With increase in longevity, the older population is increasing worldwide. The older population is more prone to the deleterious effects of renal diseases, be it an acute insult to the normal renal functions or a chronic disease. In India, the older population accounts for $7.5 \%$ of the total population.

Chronic kidney disease (CKD) patients, particularly those with end-stage renal disease (ESRD), are at much higher risk of cardiovascular disease than the general population. Cardiovascular disease is by far the leading cause of morbidity and mortality in dialysis patients, accounting for almost $40 \%$ of hospitalizations and almost $50 \%$ of deaths and, 
after stratification for age, race and gender, the cardiovascular mortality rate in ESRD patients is _10-20 times that in the general population. It is therefore not surprising that CKD has recently been called a 'vasculopathic state'. In order to prevent or at least delay the development of cardiac abnormalities, understanding the determinants of cardiovascular disease and preparing interventions aimed at correcting them is vitally important in the management of $\mathrm{CKD}$ patients..

Therefore the present study has been planned with an aim to see the cardiac finding in ckd patient in geriatric population admitted in the Department of Medicine under nephrology unit, Dr. Bhimrao Ambedkar Hospital Raipur (C.G.).

\section{Criteria}

All patients aged above 50 years admitted to the Department of Medicine under nephrology unit, Dr. Bhimrao Ambedkar Hospital Raipur (C.G.) during the period of Feb 2018 to July 2018, were included in our study. One Hundred patients with CKD were considered for the study after explaining the objectives of the study. Informed written consent was taken from all of them. The study was conducted after obtaining the approval by the Institute Ethics Committee. Patients with preexisting renal disease were also included in the study. A total of 100 patients met the above requirements and were evaluated retrospectively.

\section{Inclusion criteria}

1. Patient aged 50 years or more.

2. CKD diagnosed based on 'KDIGO 2012 Clinical Practice Guidelines for the Evaluation and Management of CKD'.

\section{Exclusion criteria}

1. Those who were not willing to participate in the study.

2. Age less than 50 years.

3. HIV-infected patients.

4. Congenital heart diseases.

5. Pulmonary obstructive and restrictive diseases.

6. Connective tissue diseases.

7. Chronic liver disease.

8. Valvular heart diseases.

9. Hypothyroidism and Hyperthyroidism.

\section{Materials and Method}

The study was carried out patient admitted in the department of medicine under nephrology unit, Dr. Bhimrao Ambedkar Hospital Raipur (C.G.) between month of February to July 2018. The records of patients of more than 50 years of age, who presented at Kidney and Dialysis Clinic and were admitted at the department of medicine under nephrology unit, Dr. Bhimrao Ambedkar Hospital Raipur (C.G.) during the above mentioned period were analysed based on Clinical evaluation and relevant investigations including echocardiography and classified under different categories of renal diseases. Ethical clearance has taken from the ethical committee of the Pt. JNM Medical College Raipur, prior to conduct the study. All participants and family members of the patients were provided written information consent.. The cut off age has been reduced to 50 years in this study to define older population due to variation in life expectancy in Indian population as compared to their western counterparts.

These patients were classified under various categories of renal diseases which included acute kidney injury (AKI), chronic kidney disease (CKD), nephrotic syndrome, obstructive uropathy and miscellaneous categories.

The history was obtained from the patients with CKD, with special reference to the symptoms of CKD and cardiac disease, risk factor for developing CKD, co-morbid conditions, duration of diagnosis of $\mathrm{CKD}$, and duration of hemodialysis. Clinical examination was done with special emphasis on signs of CKD and cardiac diseases.

Each subject underwent the following investigations: Renal function tests, liver function tests, serum electrolytes, fasting plasma glucose, postprandial plasma glucose, complete blood count, ultrasound abdomen, chest X-ray (CXR), electrocardiography (ECG), and echocardiography.

Glomerular Filtration Rate (GFR) was estimated using Cockrault-Gault formula and staging of CKD was done. USG abdomen was done to note for the size and echotexture of the kidney. It was also used to rule out hepatic disease and portal 
hypertension as they can independently affect cardiovascular system. CXR was done to rule out obstructive and restrictive lung diseases and to look for features suggestive of $\mathrm{PH}$, pulmonary edema, pleural effusion, cardiomegaly, and pericardial effusion. ECG was done to assess features of pulmonary hypertension $(\mathrm{PH})$, right ventricular (RV) strain pattern, left ventricular (LV) strain pattern, ischemic heart disease (IHD), arrhythmia, and heart blocks. Echocardiography was done in all patients to evaluate chamber size, LV systolic dysfunction, LV diastolic dysfunction, pericardial effusion, and to estimate pulmonary artery systolic pressure (PASP).

\section{Result and Analysis}

\section{Clinical Findings \& General Characterstics}

A total of 100 older patients presented between Feb 2018 and Jul 2018, out of which 76(76\%) were males and 24(24\%) were females (Table 1). Mean age was $56.62 \pm 5.44$ years.

Table (1) Demographic and clinical presentation of study patients

\begin{tabular}{|c|c|c|}
\hline Characteristics & Data & $(\%)$ \\
\hline Male & 76 & 76 \\
\hline Female & 24 & 24 \\
\hline Age & $\geq 50$ & - \\
\hline Mean & $56.62 \pm 5.44$ years & - \\
\hline Sex ratio M:F & $76: 24$ & - \\
\hline
\end{tabular}

Breathing probleme is the commonest symptom, which was present in 89 out of 100 patients i.e $89 \%$. Pedal edema was present in 79 out of 100 patients (79\%). Fatigue shows in $70(70 \%)$, Pulmonary Edema found in 50 (50\%), Chest Pain in $16(16 \%)$ out of 100 patient.
Table (2) Clinical finding of study patients

\begin{tabular}{|c|c|c|}
\hline \multicolumn{2}{|c|}{ Symptoms of patient } & $(\%)$ \\
\hline Fatigue & 70 & 70 \\
\hline Pedal Edema & 79 & 79 \\
\hline Pulmonary Edema & 50 & 50 \\
\hline Chest Pain & 16 & 16 \\
\hline Breathlessness & 89 & 89 \\
\hline
\end{tabular}

\section{Anemia in patients}

Anaemia, which is thought to make a substantial contribution to the development of cardiac abnormalities in CKD patients, is a very frequent complication. Anemia is very common in CKD and contributes to cardiovascular disease. Various factors responsible for anemia, which include erythropoietin deficiency, diminished red cell survival, deficiency of iron, Vitamin B12 and folate due to malabsorption and anorexia, impaired coagulation and platelet function due to uremia, hyperparathyroidism, bone marrow fibrosis, and chronic inflammation. In our study, anemia had very high prevalence of $93 \%$ among CKD patients.

Table (3) Anemic Episode in the study of CKD patients.

\begin{tabular}{|c|c|c|}
\hline Variable & $\begin{array}{c}\text { No. of } \\
\text { patient }\end{array}$ & $\begin{array}{c}\text { Hb less than } \\
\mathbf{1 0}-\mathbf{1 2} \text { gm } \%\end{array}$ \\
\hline CKD with HD & 80 & 78 \\
\hline CKD without HD & 20 & 15 \\
\hline $6-61$ Total & 100 & 93 \\
\hline
\end{tabular}

\section{Hypertension}

Hypertension is a frequent finding at all stages of CKD. Although its prevalence varies widely depending on the nature of the underlying renal disease, it increases nearly linearly as renal function falls, and so the vast majority of patients with significant renal failure present high blood pressure. Systolic hypertension and diastolic hypertension were present in $80(80 \%)$ and 70 (70\%) respectively.

Table (4) Systolic \& Diastolic Hypertension finding of study patients

\begin{tabular}{|c|c|c|c|}
\hline \multicolumn{2}{|c|}{ Systolic Blood Pressure $(\mathbf{n}=\mathbf{1 0 0})$} & \multicolumn{2}{c|}{ Diastolic Blood Pressure $(\mathbf{n}=\mathbf{1 0 0})$} \\
\hline Grading $(\mathbf{m m} \mathbf{H g})$ & Total & Grading $(\mathbf{m m} \mathbf{H g})$ & Total \\
\hline Normal $<120$ & 5 & Normal $<80$ & 15 \\
\hline Pre hypertension $120-139$ & 15 & Pre hypertension $80-89$ & 15 \\
\hline Stage 1 hypertension $140-159$ & 25 & Stage 1 hypertension $90-99$ & 27 \\
\hline Stage 2 hypertension $\geq 160$ & 55 & Stage 2 hypertension $\geq 100$ & 43 \\
\hline
\end{tabular}




\section{Dyslipidemia}

Lipid abnormalities are more frequent in CKD patients than in the general population, although their prevalence varies widely depending on the cause and stage of CKD. CKD-related lipid disorders mainly consist of increased serum triglyceride levels (due to an enhanced production and accumulation of triglyceriderich lipoproteins, such as very low-density lipoproteins and intermediate-density lipoproteins), low highdensity lipoprotein cholesterol levels, increasedamounts of small low-density lipoproteins, increased plasma concentrations of lipoprotein (a), and a number of qualitative changes in apolipoprotein (b) that impair the metabolism of several lipoprotein classes and thus ultimately contribute to progressive atherosclerosis.

The results of some recent studies suggest that these complex changes in lipid profiles may significantly contribute to the high cardiovascular mortality and morbidity of CKD patients.

Table (5) Trend of changes in lipids, lipoproteins, and apoA-IV in various stages of

\begin{tabular}{|c|c|c|c|}
\hline $\begin{array}{c}\text { Paramete } \\
\text { r }\end{array}$ & $\begin{array}{c}\text { CK } \\
\text { D } \\
5\end{array}$ & $\begin{array}{c}\text { Hemodialysi } \\
\text { s }\end{array}$ & $\begin{array}{c}\text { Peritonea } \\
\text { I dialysis }\end{array}$ \\
\hline $\begin{array}{c}\text { Total } \\
\text { cholesterol }\end{array}$ & $\nearrow$ & $\leftrightarrow \downarrow$ & $\uparrow$ \\
\hline $\begin{array}{c}\text { LDL } \\
\text { cholesterol }\end{array}$ & $\nearrow$ & $\leftrightarrow \downarrow$ & $\uparrow$ \\
\hline $\begin{array}{c}\text { HDL } \\
\text { cholesterol }\end{array}$ & $\downarrow$ & $\downarrow$ & $\downarrow$ \\
\hline $\begin{array}{c}\text { Non-HDL } \\
\text { cholesterol }\end{array}$ & $\nearrow$ & $\leftrightarrow \downarrow$ & $\uparrow$ \\
\hline TG & $\nearrow$ & $\uparrow$ & $\uparrow$ \\
\hline Lp (a) & $\nearrow$ & $\uparrow$ & $\uparrow \uparrow$ \\
\hline ApoA-I & $\searrow$ & $\downarrow$ & $\downarrow$ \\
\hline ApoA-IV & $\nearrow$ & $\uparrow$ & $\uparrow$ \\
\hline ApoB & $\nearrow$ & $\leftrightarrow \downarrow$ & $\uparrow$ \\
\hline
\end{tabular}

Notes: Non-HDL cholesterol includes cholesterol in LDL, VLDL, IDL, and chylomicron and its remnant. Explanation of arrows: normal $(\leftrightarrow)$, increased $(\uparrow)$, markedly increased $(\uparrow \uparrow)$, and decreased $(\downarrow)$ plasma levels compared with non- uremic individuals; increasing $(\square)$ and decreasing $(\square)$ plasma levels with decreasing GFR.

Abbreviations: apoA-IV, apolipoprotein A-IV; CKD, chronic kidney disease; LDL, low-density lipoprotein; HDL, high-density lipoprotein; TG, triglyceride; Lp (a), lipoprotein(a); ApoA-I, apolipoprotein A-I; ApoB, apolipoprotein B; VLDL, very-low-density lipoprotein; IDL, intermediate- density lipoprotein; GFR, glomerular filtration rate.

\section{Echocardiographic finding}

The Echocardiographic findings in the patient studied under shows following results. The $\mathrm{PH}$ was present in 42 patients. Pericardial effusion was present in 18 patients. HFrEF \& HFpEF was present in 32 \& 70 patients. Patients, with HFpEF being more common than HFrEF. LV hypertrophy was present in 49 patients.

Table (6) Echocardiographic findings in the patients studied

\begin{tabular}{|c|c|c|}
\hline ECHO Finding & No. of Patients & $\%$ \\
\hline PH & 42 & $42 \%$ \\
\hline Pericardial effusion & 18 & $18 \%$ \\
\hline HFrEF & 32 & $32 \%$ \\
\hline Men HFpEF & 70 & $70 \%$ \\
\hline LV Hypertrophy & 49 & $49 \%$ \\
\hline
\end{tabular}

\section{Discussion}

With increasing life expectancy, proportion of older men and women suffering from renal disorders is on the rising trend. Greater proportions of older population are now seen occupying hospital beds with problems related to their kidneys.

Out of 100 older patients with renal diseases, CKD was the commonest presentation. CKD patients have higher mortality, when compared to the general population, which is mainly attributed to cardiovascular events. Deaths due to cardiovascular events are far more common than progressing to ESRD and the need of renal replacement therapy

Proteinuria, whether considered as a marker of systemic endothelial dysfunction or a result of renal damage, has been associated with increased 
cardiovascular mortality. In repeated studies, the presence of micro- and macroalbuminuria and GFR reduction were independent predictors of increased overall and cardiovascular mortality in both diabetic patients and non-diabetic patients.

Irrespective of the presence of proteinuria, decline in GFR has been associated with increased cardiovascular morbidity and mortality. An inverse relationship between GFR and the severity of coronary artery stenosis was found as well as increased probability of having triple vessel disease with decreasing GFR.

\section{Conclusion}

Cardiovascular disease is the leading cause of morbidity and mortality in CKD patients. The significant prevalence of patients starting RRT with signs of wellestablished cardiovascular abnormalities is indirect proof that cardiovascular risk factors are present from the early stages of CKD. In this context, the early detection and treatment of modifiable risk factors must be seen as a primary challenge for the nephrologist in the everyday management of CKD patients. Particular care must be taken to give optimal treatment for the most important cardiovascular risk factors active in CKD patients., i.e. hypertension, anaemia, and lipid abnormalities.

This study concludes that substantial number of patients with CKD suffers cardiovascular disease. HFpEF is more common than HFrEF. The risk factors for $\mathrm{LV}$ failure are anemia, volume overload secondary to fluid retention, and pressure overload secondary to systemic hypertension. Pulmonary hypertension is one of the recently recognized complications of CKD which is often overlooked. All these cardiovascular manifestations independently contribute to adverse outcome in these patients. Nevertheless, it is known that, despite the high prevalence of traditional cardiovascular risk factors in CKD patients, their predictive value in terms of cardiovascular outcome is not as strong as in the general population, and that the management of well-established risk factors such as hypertension, anaemia, hyperparathyroidism and dyslipidaemia, in addition to often being inadequate, fails to prevent the excess of cardiovascular events in CKD patients. Accordingly, the management of cardiovascular risk of patients with CKD in the new millennium urgently needs adjunctive pharmacological therapies with different targets from those of more conventional approaches.

\section{References}

1. Bellomo R, Kellum J A, Ronco C. Acute kidney injury.Lancet.2012; 380:756-66.

2. Centers for Disease Control and Prevention. Leading causes of death. 2013.

3. Modi G, Jha V. Incidence of ESRD in India. Kidney Int. 2011; 79:573.

4. Pateinakis P, Papagianni A. Cardiorenal syndrome type 4-cardiovascular disease in patients with chronic kidney disease: epidemiology, pathogenesis, and management. Int $\mathrm{J}$ Nephrol. 2011; 2011:8938651. http://dx.doi.org/10.4061/2011/938651.

5. Ronco C, McCullough P, Anker S D, et al. Cardio-renal syndromes: report from the consensus conference of the acute dialysis quality initiative. Eur Heart J. 2010; 31:703-711.

6. House A A, Anand A, Bellomo R, et al. Definition and classification of Cardio-Renal Syndromes: workgroup statements from the 7th ADQI Consensus Conference. Nephrol Dial Transplant. 2010; 25:1416-1420.

7. Keith D S, Nichols G A, Gullion C M, et al. Longitudinal follow-up and outcomes among a population with chronic kidney disease in a large managed care organization. Arch Intern Med. 2004;164(March (6)):659-663.

8. Stehouwer C D A, Smulders Y M. Microalbuminuria and risk for cardiovascular disease: analysis of potential mechanisms. JASN. 2006; 17(8):2106-2111.

9. Ninomiya $T$, Perkovic V, De Galan BE, et al. Albuminuria and kidney function independently predict cardiovascular and renal outcomes in diabetes. JASN. 2009;20(8):1813-1821.

10. Astor B C, Hallan S I, Miller E R, Yeung E, Coresh J. Glomerular filtration rate, albuminuria, and risk of cardiovascular and all-cause mortality in the US population. Am J Epidemiol. 2008; 167(10):1226-1234. 
11. Weber-Mzell D, Kotanko P, Schumacher M, et al. Coronary anatomy predicts presence or absence of renal artery stenosis: a prospective study in patients undergoing cardiac catheterization for suspected coronary artery disease. Eur Heart J. 2002;23(21):1684-1691.

12. McCullough P A. Why is chronic kidney disease the "spoiler', for cardiovascular outcomes? J Am Call Cardiol. 2003;41(5):725-728.
13. Kasiske B L. The kidney in cardiovascular disease. Ann Intern Med. 2001;134:

14. Babitt J, Lin H Y. Mechanisms of Anemia in CKD. JASN. 2012;23(October): 1631-1634.

15. Artz A S, Culleton B. Prevalence of anemia in the nursing home: contribution of chronic kidney disease. J Am Geriatr Soc. 2007 ; October (10)):1566-1570. 707-709.

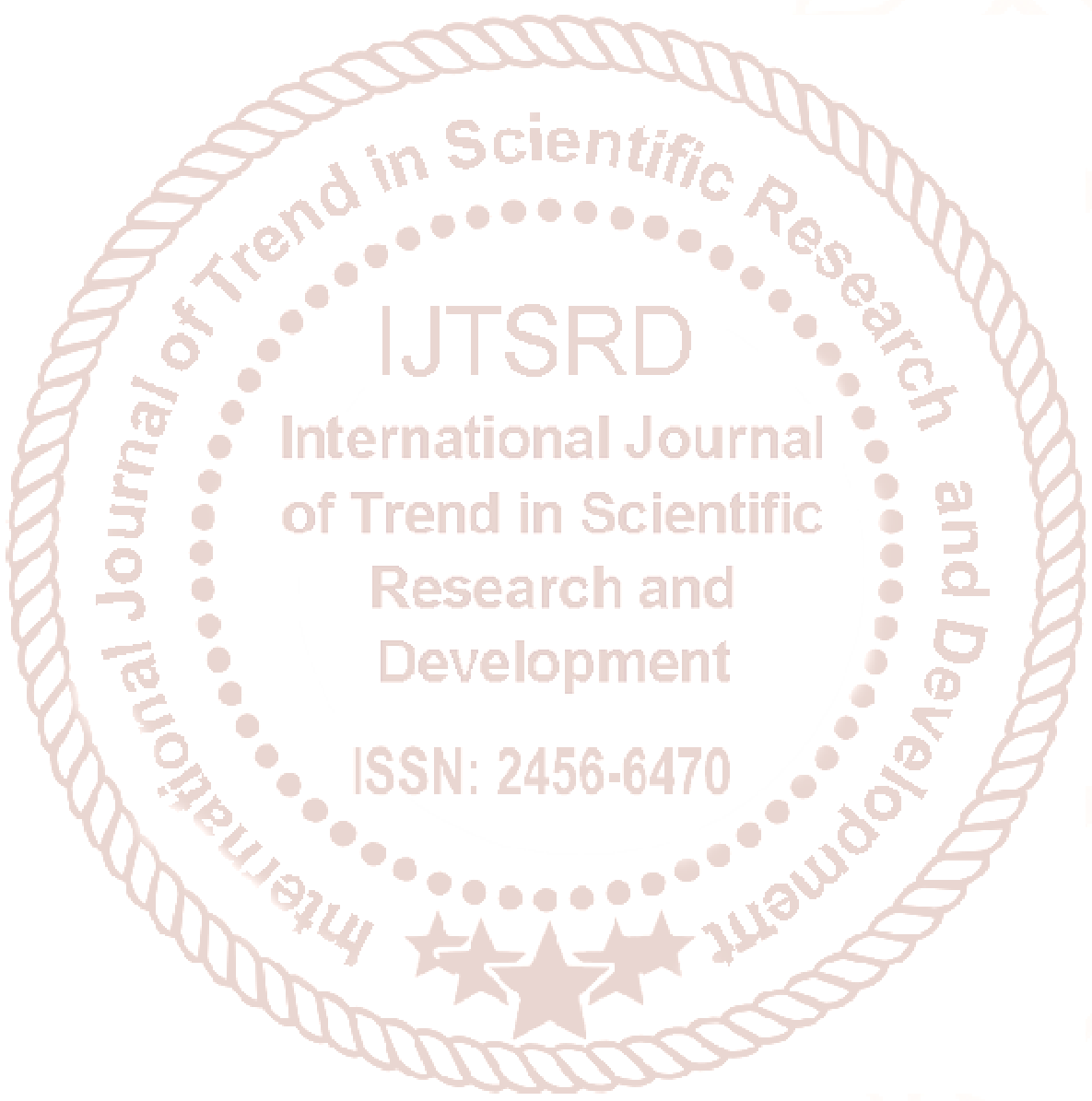

